Proceedings of the Institute of Mathematics and Mechanics,

National Academy of Sciences of Azerbaijan

Volume 46, Number 2, 2020, Pages 171-179

https://doi.org/10.29228/proc.26

\title{
ON NEGATIVE EIGENVALUES OF THE SCHRÖDINGER OPERATOR
}

\author{
ELSHAD H. EYVAZOV AND DAVUD H. ORUJOV
}

\begin{abstract}
In the paper, we consider the Schrödinger operator in an electric field, depending on the intensity of the magnetic field. Under certain constraints imposed on the electric potential, we prove its selfadjointness. It is proved that if the exact lower bound of the electric field is negative, then with an increase in the intensity of the magnetic field below the threshold of the essential spectrum, negative eigenvalues appear. We establish that as the magnetic field intensity increases, the number of negative eigenvalues of the Schrödinger operator increases.
\end{abstract}

\section{Introduction}

From Kato's magnetic inequality (see, for example, [6, p. 28]), it follows that under the influence of an external magnetic field, the energy of the system increases. For this reason, a superconducting material in a magnetic field loses its superconducting quality. We can expect that if the exact lower bound of the electric potential $V(x)$ is negative, then at sufficiently large values of the magnetic field strength $\mu$, due to the potential $\mu^{2} V(x)$ of the inductive field, the material will again restore its superconducting quality. This means that the Euler-Lagrange equation corresponding to the Ginsburg-Landau functional, i.e., Schrödinger equation

$$
-\Delta u(x)+\mu^{2} V(x) u(x)=\lambda u(x),
$$

for some negative values of the spectral parameter $\lambda$, will have a nontrivial solution from the space $L_{2}\left(R^{n}\right)$.

The objective of the paper is to give a rigorous mathematical proof of the above-mentioned heuristic reasoning.

In the space $L_{2}\left(R^{n}\right)$ we introduce the operator $H_{\mu}^{0}$ with the domain $D\left(H_{\mu}^{0}\right)=$ $C_{0}^{\infty}\left(R^{n}\right)$, operating according to the rule

$$
H_{\mu}^{0} \psi(x)=-\Delta \psi(x)+\mu^{2} V(x) \psi(x),
$$

where $\Delta$ is the Laplace operator; $C_{0}^{\infty}\left(R^{n}\right)$ is the class of infinitely differentiable complex-valued functions with compact support in $R^{n} ; \mu$ the magnetic field

2010 Mathematics Subject Classification. 35P20, 35J10, 81Q35.

Key words and phrases. Schrodinger operator, self-adjointness, essential spectrum, negative eigenvalues, magnetic field intensity. 
strength; and $V(x)$ the real function defined in the $n$-dimensional Euclidean space $R^{n}$.

We impose the following constraints on the function $V(x)$ :

a) $V(x) \in C^{2}\left(R^{n}\right)$; b) $\lim _{x \rightarrow \infty} V(x)=0 ;$ c) $\inf _{x \in R^{n}} V(x)<0$.

We denote the closure of the operator $H_{\mu}^{0}$ by $H_{\mu}$ and call it the Schrödinger operator. In the work, we establish the self-adjointness of the Schrödinger operator $H_{\mu}$ (i.e., the essentially self-adjointness of the operator $H_{\mu}^{0}$ ), study its spectrum and prove that as the magnetic field intensity $\mu$ increases below the threshold of the essential spectrum, negative eigenvalues of the operator $H_{\mu}$ appear.

Note that recently there has been great interest in the Schrödinger magnetic operator (see $[1,2,3,5,6,7,11-16]$ ). Many works are dedicated to issues of self-adjointness (see $[1,6,11]$ ), to the study of negative eigenvalues (see $[2,4,5$, $8,10,14,15]$ ), which play a key role in various fields of physics and chemistry, as well as to the study of the essential spectrum of the Schrödinger operator (see $[3,7])$.

\section{Self -Adjointness}

First, using the self-adjointness criterion (see [13, p. 283, Theorem VIII. 3]) and the Fournais-Helffer method (see [6, p. 7, Theorem 1.2.2]), we prove the essential self-adjointness of the operator $H_{\mu}^{0}$.

Theorem 2.1. Let conditions a) and b) be satisfied. Then the operator $H_{\mu}^{0}$ is essentially self-adjoint on $C_{0}^{\infty}\left(R^{n}\right)$.

Proof. From conditions a) and b) it follows that the operator $H_{\mu}^{0}$ is bounded below on $C_{0}^{\infty}\left(R^{n}\right)$. Therefore, one can find a positive number $t$ such for any function $\psi(x)$ from the space $C_{0}^{\infty}\left(R^{n}\right)$ the following inequality will hold true

$$
\left.\left(H_{\mu}^{0}+t E\right) \psi(x), \psi(x)\right) \geq\|\psi(x)\|^{2},
$$

where $E$ is the identity operator, $(\cdot, \cdot)$ and $\|\cdot\|$ the scalar product and norm, respectively, in the space $L_{2}\left(R^{n}\right)$.

Let's show that the domain of the operator $H_{\mu}^{0}+t E$ is everywhere dense in $L_{2}\left(R^{n}\right)$. Suppose that there exists a function $f(x)$ from $L_{2}\left(R^{n}\right)$ such that

$$
\left(f,\left(H_{\mu}^{0}+t E\right) \psi(x)\right)=0, \quad \forall \psi(x) \in C_{0}^{\infty}\left(R^{n}\right) .
$$

It follows from condition (2.2) that, in the sense of generalized functions, a regular generalized function $f(x)$ satisfies the equation

$$
-\Delta f(x)+\mu^{2} V(x) f(x)+t f(x)=0 .
$$

From this equality and condition a) it follows that $f(x) \in W_{2, l o c}^{2}\left(R^{n}\right)$, where $W_{2, l o c}^{2}\left(R^{n}\right)$ is the second order local Sobolev space.

Let's consider a sequence $\left\{C_{k}(x)\right\}_{k=1}^{\infty}$ from the space $C_{0}^{\infty}\left(R^{n}\right)$ elements of which are defined as follows: 


$$
C_{k}(x)=\left\{\begin{array}{cll}
0, & \text { if } & |x| \geq 2 k, \\
1, & \text { if } & |x| \leq k, \quad k=1,2, \ldots, \\
\text { sufficiently smooth, } & \text { if } & k \leq|x| \leq 2 k,
\end{array}\right.
$$

where $|x|=\sqrt{x_{1}^{2}+x_{2}^{2}+\ldots+x_{n}^{2}}$.

It is evident that

$$
\lim _{k \rightarrow \infty} C_{k}(x)=1 \text { (pointwise) }
$$

and

$$
\lim _{k \rightarrow \infty} \nabla C_{k}(x)=0 \quad \text { (pointwise) }
$$

where $\nabla=\left(\frac{\partial}{\partial x_{1}}, \frac{\partial}{\partial x_{2}}, \ldots, \frac{\partial}{\partial x_{n}}\right)$.

Using the Green's formula, we calculate the integral

$$
\int_{R^{n}}\left[-\Delta\left(C_{k}^{2}(x) u(x)\right)\right] \overline{f(x)} d x
$$

where $u(x) \in C_{0}^{\infty}\left(R^{n}\right)$. We then have:

$$
\begin{aligned}
& \int_{R^{n}}\left[-\Delta\left(C_{k}^{2}(x) u(x)\right)\right] \overline{f(x)} d x=-\sum_{j=1}^{n} \int_{R^{n}} \frac{\partial^{2}\left(C_{k}^{2}(x) u(x)\right)}{\partial x_{j}^{2}} \overline{f(x)} d x= \\
= & -\sum_{j=1}^{n} \int_{R^{n}} \frac{\partial}{\partial x_{j}}\left[\frac{\partial\left(C_{k}^{2}(x) u(x)\right)}{\partial x_{j}^{2}} \overline{f(x)}\right] d x+\sum_{j=1}^{n} \int_{R^{n}} \frac{\partial\left(C_{k}^{2}(x) u(x)\right)}{\partial x_{j}} \frac{\partial \overline{f(x)}}{\partial x_{j}} d x= \\
= & \sum_{j=1}^{n} \int_{R^{n}} \frac{\partial\left(C_{k}^{2}(x) u(x)\right)}{\partial x_{j}} \frac{\partial \overline{f(x)}}{\partial x_{j}}=2 \sum_{j=1}^{n} \int_{R^{n}} C_{k}(x) \frac{\partial C_{k}(x)}{\partial x_{j}} \frac{\partial \overline{f(x)}}{\partial x_{j}} u(x) d x+ \\
+\sum_{j=1}^{n} \int_{R^{n}} C_{k}^{2}(x) \frac{\partial u(x)}{\partial x_{j}} \frac{\partial \overline{f(x)}}{\partial x_{j}}= & 2 \int_{R^{n}} C_{k}(x)\left[\nabla C_{k}(x) \cdot \nabla \overline{f(x)}\right] u(x) d x+2 \int_{R^{n}} C_{k}^{2}(x)[\nabla \overline{f(x)} \cdot \nabla u] d x .
\end{aligned}
$$

A simple calculation shows that the following formula holds true:

$$
\begin{gathered}
\left.\int_{R^{n}} \nabla C_{k}(x) \overline{f(x)} \cdot \nabla\left(C_{k}(x) u(x)\right) d x=\int_{R^{n}}\left(\nabla C_{k}(x)\right)^{2} \overline{f(x}\right) u(x) d x+ \\
+\int_{R^{n}} C_{k}(x) \nabla C_{k}(x) \cdot[\overline{f(x)} \nabla u(x)-u(x) \nabla \overline{f(x)}] d x+ \\
+2 \int_{R^{n}} C_{k}(x) \nabla C_{k}(x) \cdot \nabla \overline{f(x)} u(x) d x+\int_{R^{n}} C_{k}^{2}(x) \nabla \overline{f(x)} \cdot \nabla u(x) d x .
\end{gathered}
$$

By adding to each side of equality (2.7) the integral 


$$
\int_{R^{n}}\left(\mu^{2} V(x)+t\right) C_{k}^{2}(x) \overline{f(x)} u(x) d x
$$

and taking into account equalities (2.3) and (2.6), we obtain

$$
\begin{aligned}
& \left.\int_{R^{n}} \nabla\left(C_{k}(x) \overline{f(x)}\right) \cdot \nabla\left(C_{k}(x) u(x)\right) d x+\int_{R^{n}}\left[\mu^{2} V(x)+t\right] C_{k}^{2}(x) \overline{f(x}\right) u(x) d x= \\
& \left.=\int_{R^{n}}\left(\nabla C_{k}(x)\right)^{2} \overline{f(x}\right) u(x) d x+\int_{R^{n}} C_{k}(x) \nabla C_{k}(x) \cdot[2 i \operatorname{Im}(\overline{\mathrm{f}(\mathrm{x})} \nabla \mathrm{u}(\mathrm{x}))] d x .
\end{aligned}
$$

It is evident that $f(x) \in W_{2, l o c}^{1}\left(R^{n}\right)$, as $f(x) \in W_{2, l o c}^{2}\left(R^{n}\right)$. From everywhere density of $C_{0}^{\infty}\left(R^{n}\right)$ in $W_{2, l o c}^{1}\left(R^{n}\right)$ it follows that equality (2.8) holds true even for $u(x)=f(x)$. Substituting the function $f(x)$ into $u(x)$ in equality (2.8), we obtain

$$
\begin{aligned}
& \int_{R^{n}} \mid \nabla\left(\left.f(x) C_{k}(x)\right|^{2} d x+\int_{R^{n}}\left[\mu^{2} V(x)+t\right] C_{k}^{2}(x)|f(x)|^{2} d x=\right. \\
= & \left.\int_{R^{n}}\left(\nabla C_{k}(x)\right)^{2}|f(x)|^{2} d x+\int_{R^{n}} C_{k}(x) \nabla C_{k}(x)[2 i \operatorname{Im}(\overline{\mathrm{f}(\mathrm{x}}) \nabla \mathrm{f}(\mathrm{x}))\right] d x .
\end{aligned}
$$

Hence we have

$$
\begin{gathered}
\int_{R^{n}}\left|\nabla\left(f(x) C_{k}(x)\right)\right|^{2} d x+\int_{R^{n}}\left[\mu^{2} V(x)+t\right] C_{k}^{2}(x)|f(x)|^{2} d x= \\
=\int_{R^{n}}\left(\nabla C_{k}(x)\right)^{2}|f(x)|^{2} d x .
\end{gathered}
$$

From inequality (2.1) we obtain:

$$
\begin{array}{r}
\left\|C_{k}(x) f(x)\right\|^{2} \leq\left(\left(H_{\mu}^{0}+t E\right) C_{k}(x) f(x), C_{k}(x) f(x)\right)= \\
=\int_{R^{n}}\left|\nabla\left(f(x) C_{k}(x)\right)\right|^{2} d x+\int_{R^{n}}\left[\mu^{2} V(x)+t\right] C_{k}^{2}(x)|f(x)|^{2} d x .
\end{array}
$$

Using equality (2.9), inequality (2.10) can be rewritten as follows:

$$
\left\|C_{k}(x) f(x)\right\|^{2} \leq \int_{R^{n}}\left(\nabla C_{k}(x)\right)^{2}|f(x)|^{2} d x .
$$

From the properties (2.4) and (2.5) of the sequence $\left\{C_{k}(x)\right\}_{k=1}^{\infty}$ and inequality (2.11) we obtain:

$$
\begin{aligned}
\|f(x)\|^{2} & =\lim _{k \rightarrow \infty}\left\|C_{k}(x) f(x)\right\|^{2} \leq \varlimsup_{k \rightarrow \infty}\left\|C_{k}(x) f(x)\right\|^{2} \leq \\
& \leq \varlimsup_{k \rightarrow \infty} \int_{R^{n}}\left(\nabla C_{k}(x)\right)^{2}|f(x)|^{2} d x=0 .
\end{aligned}
$$

Hence we have that $f(x)=0$ almost everywhere in $R^{n}$. Therefore, the operator $H_{\mu}^{0}+t E$ is an essentially self-adjoint operator. It follows that the operator $H_{\mu}^{0}$ 
is essentially self-adjoint on $C_{0}^{\infty}\left(R^{n}\right)$, as $t E$ is the bounded self-adjoint operator. This completes the proof of the theorem.

From theorem II .4 of [9] we get that the following lemma is true

Lemma 2.1. If conditions a) and b) are satisfied, then the essential spectrum of the Schrödinger operator $H_{\mu}$ coincides with the positive half-axis, i.e.,

$$
\sigma_{e s s}\left(H_{\mu}\right)=[0,+\infty)
$$

\section{Proof of the main result}

Now we show that as the magnetic field strength $\mu$ increases below the threshold of the essential spectrum, i.e., below zero, the eigenvalues of the operator $H_{\mu}$ appear.

Theorem 3.1. Let the function $V(x)$ satisfy conditions a)-c). Then, for sufficiently large $\mu$, the Schrödinger operator $H_{\mu}$ has at least one negative eigenvalue. Proof. It is known that (see $\left[6\right.$, p. 9]) the ground state of the operator $H_{\mu}$ is defined as follows:

$$
\inf \sigma\left(H_{\mu}\right)=\inf _{u(x) \in W_{2}^{1}\left(R^{n}\right) \backslash\{0\}} \frac{\int_{R^{n}}\left\{|\nabla u(x)|^{2}+\mu^{2} V(x)|u(x)|^{2}\right\} d x}{\int_{R^{n}}|u(x)|^{2} d x} .
$$

Note that the quantity (3.1) is important in classical quantum mechanics (see [16]), since it is the lowest energy that a particle can reach in $R^{n}$, interacting with the force field generated by the potential $\mu^{2} V(x)$ (and which will ultimately be achieved due to radiation of energy).

To prove the theorem, it suffices to prove that there exists a function $\varphi_{\mu}(x)$ from $W_{2}^{1}\left(R^{n}\right) \backslash\{0\}$ such that

$$
\frac{\int_{R^{n}}\left\{\left|\nabla \varphi_{\mu}(x)\right|^{2}+\mu^{2} V(x)\left|\varphi_{\mu}(x)\right|^{2}\right\} d x}{\int_{R^{n}}\left|\varphi_{\mu}(x)\right|^{2} d x}<0 .
$$

To prove inequality (3.2), we follow the path indicated in Example 1.3.2 of [6]. Let the function $V(x)$ reach its minimum at the point $x^{(0)}=\left(x_{1}^{(0)}, x_{2}^{(0)}, \ldots, x_{n}^{(0)}\right)$, i.e.

$$
\inf _{x \in R^{n}} V(x)=\min _{x \in R^{n}} V(x)=V\left(x^{(0)}\right) .
$$

Consider the function

$$
\varphi_{\mu}(x)=\bar{e}^{\mu\left|x-x^{(0)}\right|^{2}} .
$$

Let us prove that

$$
\lim _{\mu \rightarrow+\infty} \frac{\int_{R^{n}}\left\{\left|\nabla \varphi_{\mu}(x)\right|^{2}+\mu^{2} V(x)\left|\varphi_{\mu}(x)\right|^{2}\right\} d x}{\int_{R^{n}}\left|\varphi_{\mu}(x)\right|^{2} d x}=V\left(x^{(0)}\right) .
$$


Passing to the polar coordinate system, we obtain:

$$
\int_{R^{n}}\left|\varphi_{\mu}(x)\right|^{2} d x=\int_{R^{n}} \bar{e}^{2 \mu\left|x-x^{(0)}\right|^{2}} d x=\sigma_{n} \int_{R^{n}}^{+\infty} \bar{e}^{2 \mu r^{2}} r^{n-1} d r=\frac{\sigma_{n} A_{n}}{(2 \mu)^{\frac{n}{2}}},
$$

where $\sigma_{n}$ is the area of $(n-1)$-dimensional unit sphere,

$$
A_{n}=\int_{0}^{+\infty} \bar{e}^{s^{2}} s^{n-1} d s
$$

In order to calculate the integral

$$
\int_{R^{n}}\left|\nabla \varphi_{\mu}(x)\right|^{2} d x
$$

we find the partial derivatives $\frac{\partial \varphi_{\mu}(x)}{\partial x_{i}}, \quad i=1,2, \ldots, n$.

We have :

$$
\frac{\partial \varphi_{\mu}(x)}{\partial x_{i}}=\frac{\partial}{\partial x_{i}}\left(\bar{e}^{2 \mu\left|x-x^{(0)}\right|^{2}}\right)=-2 \mu e^{-\mu\left|x-x^{(0)}\right|^{2}}\left(x_{i}-x_{i}^{(0)}\right), i=1,2, \ldots, n .
$$

From here we obtain

$$
\left|\nabla \varphi_{\mu}(x)\right|^{2} d x=4 \mu^{2} e^{-2 \mu\left|x-x^{(0)}\right|^{2}}\left|x-x^{(0)}\right|^{2} d x .
$$

Using (3.5) we have:

$$
\begin{gathered}
\int_{R^{n}}\left|\nabla \varphi_{\mu}(x)\right|^{2} d x=\int_{R^{n}} 4 \mu^{2}\left|x-x^{(0)}\right|^{2} e^{-2 \mu\left|x-x^{(0)}\right|^{2}} d x= \\
=4 \mu^{2} \int_{R^{n}} \bar{e}^{2 \mu|t|^{2}}|t|^{2} d t=4 \mu^{2} \sigma_{n} \int_{0}^{+\infty} e^{-2 \mu r^{2}} r^{n+1} d r=\frac{\sigma_{n} B_{n}}{(2 \mu)^{\frac{n-2}{2}}},
\end{gathered}
$$

where

$$
B_{n}=\int_{0}^{+\infty} \bar{e}^{s^{2}} s^{n+1} d s
$$

From (3.4) and (3.6) we obtain

$$
\begin{gathered}
\frac{\int_{R^{n}}\left\{\left|\nabla \varphi_{\mu}(x)\right|^{2}+\mu^{2} V(x)\left|\varphi_{\mu}(x)\right|^{2}\right\} d x}{\int_{R^{n}}\left|\varphi_{\mu}(x)\right|^{2} d x}= \\
=\frac{\frac{\sigma_{n} B_{n}}{(2 \mu)^{\frac{n-2}{2}}}+\mu^{2} \int_{R^{n}} V(x) \bar{e}^{2 \mu\left|x-x^{(0)}\right|^{2}} d x}{\frac{\sigma_{n} A_{n}}{(2 \mu)^{\frac{n}{2}}}} .
\end{gathered}
$$

Using the Taylor formula for the function $V(x)$ at the minimum point $x^{(0)}$ with a remainder in the Lagrange form, we have: 


$$
\begin{gathered}
\int_{R^{n}} V(x) \bar{e}^{2 \mu\left|x-x^{(0)}\right|^{2}} d x=\int_{R^{n}} V\left(x^{(0)}\right) \bar{e}^{2 \mu\left|x-x^{(0)}\right|^{2}} d x+ \\
+\frac{1}{2} \sum_{i, j=1}^{n} \int_{R^{n}} \bar{e}^{2 \mu\left|x-x^{(0)}\right|^{2}} \frac{\partial^{2} V\left(\xi(x), x^{(0)}\right)}{\partial x_{i} \partial x_{j}}\left(x_{i}-x_{i}^{(0)}\right)\left(x_{j}-x_{j}^{(0)}\right) d x .
\end{gathered}
$$

From the boundedness of the function $\frac{\partial^{2} V\left(\xi(x), x^{(0)}\right)}{\partial x_{i} \partial x_{j}}$ in $R^{n}$, it follows that there exists a continuous and bounded function $M(x)$ in $R^{n}$ such that the following equality is true:

$$
\frac{1}{2} \sum_{i, j=1}^{n} \frac{\partial^{2} V\left(\xi(x), x^{(0)}\right)}{\partial x_{i} \partial x_{j}}\left(x_{i}-x_{i}^{(0)}\right)\left(x_{j}-x_{j}^{(0)}\right)=M(x)\left|x-x^{(0)}\right|^{2} .
$$

From $(3.4),(3.7),(3.8)$ and (3.9) we obtain

$$
\begin{gathered}
\frac{\int_{R^{n}}\left\{\left|\nabla \varphi_{\mu}(x)\right|^{2}+\mu^{2} V(x)\left|\varphi_{\mu}(x)\right|^{2}\right\} d x}{\int_{R^{n}}\left|\varphi_{\mu}(x)\right|^{2} d x}= \\
=\frac{\frac{\sigma_{n} B_{n}}{(2 \mu)^{\frac{n-2}{2}}}+\mu^{2} V\left(x^{(0)}\right) \frac{\sigma_{n} A_{n}}{(2 \mu)^{\frac{n}{2}}}+\mu^{2} \int_{R^{n}} \bar{e}^{2 \mu\left|x-x^{(0)}\right|^{2}} M(x)\left|x-x^{(0)}\right|^{2} d x}{\frac{\sigma_{n} A_{n}}{(2 \mu)^{\frac{n}{2}}}} .
\end{gathered}
$$

From the boundedness and continuity of the function $M(x)$ in $R^{n}$, it follows that there exists a positive number $M_{0}$ such that

$$
\int_{R^{n}} \bar{e}^{2 \mu\left|x-x^{(0)}\right|^{2}} M(x)\left|x-x^{(0)}\right|^{2} d x=M_{0} \int_{R^{n}} \bar{e}^{2 \mu\left|x-x^{(0)}\right|^{2}}\left|x-x^{(0)}\right|^{2} d x .
$$

As

$$
\int_{R^{n}} \bar{e}^{2 \mu\left|x-x^{(0)}\right|^{2}}\left|x-x^{(0)}\right|^{2} d x=\frac{\sigma_{n} B_{n}}{(2 \mu)^{\frac{n+2}{2}}},
$$

then from (3.10) and (3.11) we obtain

$$
\frac{\int_{R^{n}}\left\{\left|\nabla \varphi_{\mu}(x)\right|^{2}+\mu^{2} V(x)\left|\varphi_{\mu}(x)\right|^{2}\right\} d x}{\int_{R^{n}}\left|\varphi_{\mu}(x)\right|^{2} d x}=\mu^{2} \frac{\frac{2 B_{n}}{\mu}+A_{n} V\left(x^{(0)}\right)+\frac{M_{0} B_{n}}{2 \mu}}{A_{n}} .
$$

It follows from (3.12) that

$$
\lim _{\mu \rightarrow \infty} \frac{1}{\mu^{2}}\left\{\frac{\int_{R^{n}}\left\{\left|\nabla \varphi_{\mu}(x)\right|^{2}+\mu^{2} V(x)\left|\varphi_{\mu}(x)\right|^{2}\right\} d x}{\int_{R^{n}}\left|\varphi_{\mu}(x)\right|^{2} d x}\right\}=V\left(x^{(0)}\right) .
$$


As $V\left(x^{(0)}\right)<0$ then it follows from (3.13) that for sufficiently large $\mu$ the following inequality holds true:

$$
\frac{\int_{R^{n}}\left\{\left|\nabla \varphi_{\mu}(x)\right|^{2}+\mu^{2} V(x)\left|\varphi_{\mu}(x)\right|^{2}\right\} d x}{\int_{R^{n}}\left|\varphi_{\mu}(x)\right|^{2} d x}<0 .
$$

This completes the proof of the theorem.

Corollary 3.1. From this theorem and from the minimax principle (see [6] or [10]), it follows that as the magnetic field strength $\mu$ increases, the number of negative eigenvalues of the operator $H_{\mu}$ increases.

\section{References}

[1] A. R. Aliev, E. Kh. Eivazov, Essential self-adjointness of the Schrödinger operator in a magnetic field, Theoret. and Math. Phys., 166(2011) no.2, 228-233 (translated from Teoret. Mat. Fiz., 166, (2011), no. 2, 266-271).

[2] A.R. Aliev, E.H. Eyvazov, On the discreteness of the spectrum of the magnetic Schrödinger operator, Funct. Anal. Appl. 46 (2012), no.4, 305-307 (translated from Funktsional. Anal. i Prilozhen. 46 (2012), no. 4, 83-85).

[3] A.R.Aliev, E.H.Eyvazov, On the essential spectrum of electromagnetic Schrödinger operator with singular electric potential, Complex Variables and Elliptic Equations, 59(2014), no. 1, 18-27.

[4] T.Ekholm, R.L.Frank, On Lieb-Thirring inequalities for Schrödinger operators with virtual level, Comm. Math. Phys., 264(2006), no.3, 725-740.

[5] E.H.Eyvazov, On negative spectrum of two-dimensional Schrodinger operator in electromagnetic field, Proceedings of IMM of NAS Azerb., Special Issue - In memory of Professor M.G.Gasymov on his 75th birthday, 40(2014), 143-151.

[6] S. Fournais, B. Helffer, Spectral methods in surface superconductivity, Progress in Nonlinear Differential Equations and their Applications, 77, Birkhauser Boston Inc., Boston, MA, 2010.

[7] A.Kachmar, M.Persson, On the essential spectrum of magnetic Schrödinger operators in exterior domains, Arab Journal of Mathematical Sciences, 19(2013), no. 2, 217-222.

[8] M.Karuhang, Eigenvalue Bounds for a Class of Schrödinger Operators in a Strip, Journal of Mathematics, 2018 (2018), Article ID 7172356, 8 pages.

[9] D.Krejcirik, Schrödinger operators and their spectra, BCAM, Bilbao, (2010).

[10] A.Laptev, M.Solomyak, On the negative spectrum of two dimensional Schrödinger operator with radial potential, Comm. Math, Phys., 314 (2012), no. 1, 229-241.

[11] A.Mohamed, G.Raikov. On the Spectral Theory of the Schrödinger Operator with Electromagnetic Potential, In: Pseudo-Differential Calculus and Mathematical Physics, Berlin: Akademie Verlag, (1994), 298-390.

[12] Rajabov Sh. Sh. Generalized Dirichlet problems for magnetic Schrödinger operator, Proceedings of the Institute of Mathematics and Mechanics, National Academy of Sciences of Azerbaijan, 45(2019), no. 1, 111-118.

[13] M. Reed and B. Simon, Methods of Modern Mathematical Physics, 1: Functional Analysis, Academic Press, New York-London, 1972.

[14] G.V.Rozenbljum, Distribution of the discrete spectrum of singular differential operators, Doklady Akademii Nauk SSSR, 202 (1972), 1012-1015. 
[15] Z.Shen, On the number of negative eigenvalues for a Schrödinger operator with magnetic field., Comm. Math. Phys., 182 (1996), no.3, 637-660.

[16] E.J.M. Veling, Lower bounds for the infimum of the spectrum of the Schrödinger operator in $R^{n}$ and the Sobolev inequalities, Journal of Inequalities in Pure and Applied Mathematics, 3(2002), no.4, 1-22.

Elshad H. Eyvazov

Baku State University, Baku, Azerbaijan

Institute of Mathematics and Mechanics, National Academy of Sciences of Azerbaijan, Baku, Azerbaijan

Baku Engineering University, Baku, Azerbaijan

E-mail address: eyvazovelshad@gmail.com

Davud H. Orujov

Baku State University, Baku, Azerbaijan

E-mail address: dorudjov@ gmail.com

Received: October 9, 2019; Revised: January 30, 2020; Accepted: May 6, 2020 